\title{
Epidemiology of Fragility Fractures and Social Impact
}

\author{
Nicola Veronese, Helgi Kolk, and Stefania Maggi
}

\section{$2.1 \quad$ Introduction}

Ageing is becoming the next public health challenge worldwide. According to WHO data, the proportion of the population more than 60 years will nearly double from $12 \%$ in 2015 to $22 \%$ in 2050 . By 2050 , this population age group is expected to increase from the current 900 million to 2 billion, $80 \%$ of these people living in low- and middle-income countries [1]. Understanding the trends of age-related diseases has an important role in healthcare policy. Musculoskeletal disorders are among the most common problems affecting the elderly, with osteoporosis and osteoporotic fractures leading the field [2].

This chapter is a component of Part 1: Background.

For an explanation of the grouping of chapters in this book, please see Chapter 1: 'The Multidisciplinary Approach to Fragility Fractures Around the World-An Overview'.

\footnotetext{
N. Veronese

National Research Council, Neuroscience Institute, Aging Branch, Padova, Italy

Geriatric Unit, Department of Internal Medicine and Geriatrics, University of Palermo, Palermo, Italy

H. Kolk

Geriatric Unit, Department of Internal Medicine and Geriatrics, University of Palermo, Palermo, Italy

Department of Traumatology and Orthopaedics, University of Tartu, Tartu, Estonia e-mail: helgi.kolk@kliinikum.ee

S. Maggi $(\triangle)$

National Research Council, Neuroscience Institute, Aging Branch, Padova, Italy e-mail: stefania.maggi@in.cnr.it 
Osteoporosis, defined by WHO as bone densitometry (DXA) T-scores less than -2.5 at the lumbar spine or femoral neck and microarchitectural deterioration of bone tissue, is recognised as a major public health issue through its association with fragility fractures, particularly of the hip, vertebrae, wrist and upper arm. Sarcopenia is generally understood as an age-dependent decline in muscle mass combined with low muscle strength and/or low physical performance. The combination of osteoporosis and sarcopenia is known as osteosarcopenia, which contributes to frailty, poor balance, falls and fragility fractures [3]. Fragility fractures have been defined as fractures that occur after minimal trauma (falling from a standing height or less) or without considerable trauma. Several factors contribute to the development of fragility fractures, two major groups are those affecting/decreasing bone mineral density (BMD) and increasing the rate of falls. To provide comprehensive care to older adults, particularly with attention to musculoskeletal health, clinicians must assess and manage different aspects, including osteosarcopenia and falls.

Please refer to Chaps. 3 and 15 for the management of osteoporosis in older patients and to Chap. 16 for the prevention of falls.

Fragility fractures may have a negative impact on the quality of life of older people and their families due to loss of independence and may also lead to disability and death. Significant geographic variation occurs in the age-specific incidence in osteoporotic fractures worldwide (Fig. 2.1), with Western populations (Europe, North America and Oceania) reporting an increase in hip fracture incidence during the second half of the twentieth century and a decline since 2000 [4, 5]. However, even in these countries, the fall in age-specific incidence is far outweighed by the ageing demographic described earlier, so population incidence continues to rise [6]. Osteoporosis and sarcopenia are frequently underdiagnosed and thus undertreated, even persons with proven bone mineral density loss and previous fragility fracture do not receive appropriate treatment to prevent

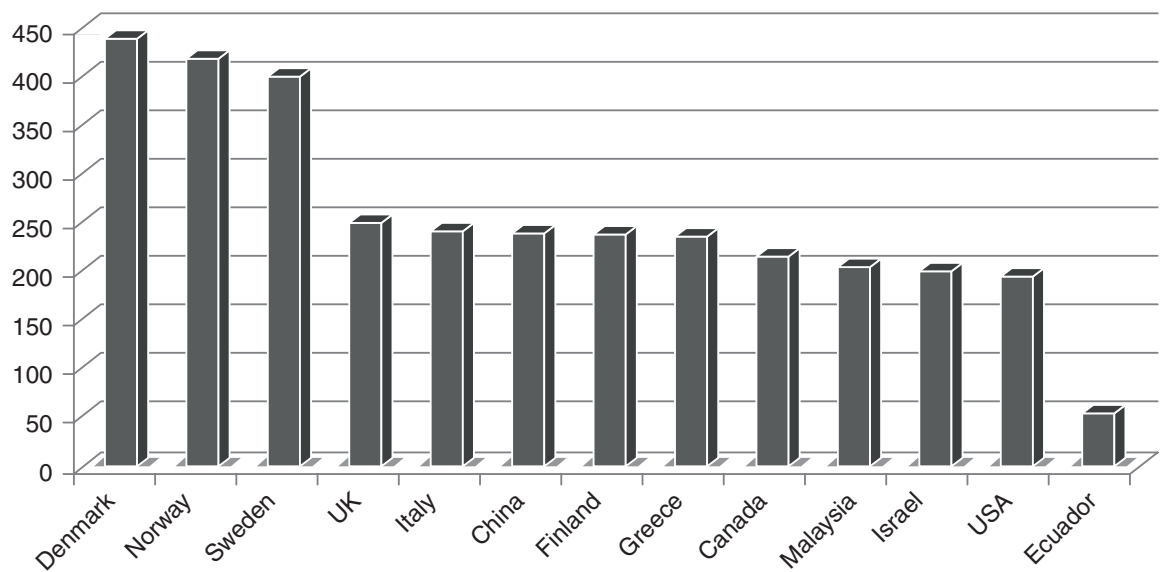

Fig. 2.1 Age-standardised hip fracture incidence rates $(/ 100,000)$ for some representative countries 
subsequent fractures. On the other hand, there is much current debate on what is the target population that may potentially benefit from treatments acting on bone metabolism [6].

The total costs of osteoporosis are difficult to calculate as cost estimates are based on many assumptions, making a comparison between countries and different healthcare systems challenging. In addition to direct medical costs in acute care of osteoporotic fractures and medications, these costs also include work-absence of family caregivers and long-term care of elderly fracture patients. The economic impact of hip fractures, the most detrimental of osteoporotic fractures, has been widely investigated, however, few studies have utilised the same instruments. Recovery from a fragility fracture can be influenced by social factors but might itself influence social activity and family relationships [7].

The objective of this chapter is to review and describe the epidemiology of osteoporosis and fragility fractures, highlighting the costs to society and the individual.

\subsection{Prevalence of Osteoporosis}

The WHO Osteoporosis Working Group and other international osteoporosis organisations have stated that the femoral neck is the only site that should be used in the estimation of osteoporosis prevalence at a population level. Osteoporosis is diagnosed using dual-energy X-ray absorptiometry (DXA), which measures bone mineral density (BMD) of the femoral neck. BMD is one of the most used tools for the management of osteoporosis, being used for diagnosis, fracture risk assessment, selection of patients for treatment and treatment monitoring [2]. According to the criterion set by a WHO Working Group, osteoporosis is diagnosed when the BMD at the femoral neck is 2.5 standard deviations or more below the average BMD of the young white female population. Accurate estimates of the prevalence of osteoporosis require country-specific data on the distribution of femoral neck BMD, for most studies, it is assumed that the mean femoral neck BMD is similar between countries at the age of 50 years and also the bone loss is similar [2]. Based on these estimates approximately 5.5 million osteoporotic men and more than 22 million osteoporotic women resided in the EU27 in 2010, that is, there were four times as many women with osteoporosis as there were men. Of all countries in the EU27, Germany was estimated to have the highest number of individuals with osteoporosis with approximately 1 million osteoporotic men and 4 million osteoporotic women. Overall the prevalence of osteoporosis was $6.6 \%$ and $22.1 \%$ in men and women aged 50 years or more and $5.5 \%$ in the general population of the EU. In males aged 50 years or more, the prevalence of osteoporosis varied from 5.7\% in Slovakia to $6.9 \%$ in Greece, Italy and Sweden. The corresponding data for females were $19.3 \%$ in Bulgaria and $23.4 \%$ in Italy [2]. The prevalence of osteoporosis increased progressively with age, although the absolute number of individuals with osteoporosis increases less markedly. When stratifying the population between ages 50 and 80 in 5-year age groups, the highest number of women with osteoporosis (approximately 3.9 million) was observed in the 75-79 year age group, for men the highest estimated number was in the age group of 60-64 years (approximately 0.8 million) [2]. 
According to a review published in 2016, in East Asia (Hong Kong, Japan, Macau, Mongolia, North Korea, the People's Republic of China, South Korea and Taiwan) osteoporosis is recognised as a growing problem because of the rapidly increasing number of older people [8]. China has the largest aged population in the world, as the population aged more than 60 years will reach 400 million (approximately $30 \%$ of the total population) by 2050 . In Japan, the percentage of the population aged more than 65 years rose from $10.3 \%$ in 1985 to $20.1 \%$ in 2005; this percentage is expected to double by 2050 [8]. Studies from the Chinese mainland, Hong Kong and Taiwan published between 1990 and 2017 have reported various prevalence rates of osteoporosis due to different reference values (US and Asian) and age groups; a clear tendency of increase in prevalence with age was recorded with osteoporosis affecting more than one-third of people aged 50 years and older. Variable osteoporosis prevalence was reported across Chinese cities before 2002: Jilin $(15.5 \%)>$ Shanghai $(14.2 \%)>$ Sichuan $(11.3 \%)>$ Guangzhou $(10.2 \%)>$ Beijing (5.2\%). According to studies by Zhang et al., with a cut-off value of $-2.5 \mathrm{SD}$, osteoporosis prevalence increased from $6.37 \%$ in women aged $40-50$ years to $76.74 \%$ in those aged 80-90 years [9]. A higher prevalence of osteoporosis was demonstrated in the female population and residents of northern China [9].

In Hong Kong, osteoporosis prevalence in women aged more than 50 years was $16 \%$ at the total hip, while in men the figure was $6 \%$ in 2005 . In earlier studies (published in 1999) the prevalence of osteoporosis in Hong Kong was significantly higher than in the mainland at the same time. The prevalence of osteoporosis in Taiwan was comparable to that in the Chinese mainland [9].

The data on the prevalence of osteoporosis among women in India come from studies conducted in small groups spread across the country. The estimates suggested that out of the 230 million Indians expected to be over the age of 50 years in $2015,20 \%$, that is, 46 million were women with osteoporosis. Thus, osteoporosis is a major public health problem in Indian women [10].

The reported prevalence of osteoporosis among postmenopausal women in Brazil varies from $15 \%$ to $33 \%$, depending on the study methodology and the use of bone densitometry data or self-reporting by participants. However, recent studies using DXA report similar prevalence data to other countries [11].

Prevalence estimates of osteoporosis or low bone mass at the femoral neck or lumbar spine (adjusted by age, sex, and race/ethnicity to the 2010 Census, defined by WHO criteria) for the non-institutionalised population age 50 years and older were applied to determine the total number of older US residents with osteoporosis and low bone mass [12]. There were over 99 million adults 50 years and older in the US in 2010. Based on an overall $10.3 \%$ prevalence of osteoporosis, the estimated number of older adults having osteoporosis was 10.2 million in 2010, women and non-Hispanic Whites having the largest counts. The prevalence of osteoporosis was higher in men $80+(10.9 \%)$ compared to men $50-59$ years of age $(3.4 \%)$; however, due to the decrease in male population counts by age, the estimated number of men with osteoporosis was lower by age: 0.7 million in the 50-59 group and 0.4 million in the $80+$ group [12]. 


\subsection{Factors Affecting Bone Mineral Density}

Non-modifiable factors negatively affecting BMD and bone microstructure include older age, female sex, White race, personal and parental history of osteoporosis and fractures, and low body frame size. The known modifiable risk factors for osteoporosis include low calcium intake, reduced exposure to sunlight, prolonged immobility, excessive alcohol intake, smoking, eating disorders, long time immobility, low body mass index (BMI) and low physical activity. Several medications (glucocorticoids, PPI, anticonvulsants, chemotherapy of breast and prostatic cancer) require special attention: if these cannot be avoided, the length of treatment courses should be modified and measures to prevent the progression of osteoporosis need to be taken. The same risk factors are generally included in the FRAX model for the assessment of fracture probability $[2,13]$.

\subsection{Osteosarcopenia}

Prevalence rates of sarcopenia vary greatly due to different definitions, tools of diagnosis, and patient populations. Prevalence rates utilising the European Working Group on Sarcopenia in Older People (EWGSOP) definition vary from 1 to $29 \%$ in elderly community-dwelling populations and from 14 to $33 \%$ in long-term care populations [14]. Advanced age consistently appears to be a risk factor for sarcopenia. In most studies that reported gender, there was no significant association with sarcopenia prevalence. Nursing home residents, patients with hip fracture and these more than 80 years have higher rates of diagnosis [14].

Epidemiological measures of osteosarcopenia are fairly limited due to the recent origin of the term. A study of 680 elderly adults with a history of falls found an osteosarcopenia prevalence of $37 \%$, with these patients having a higher frequency of comorbidities, impaired mobility and depression [15].

\subsection{Falls}

Age is one of the key risk factors for falls. Older people have the highest risk of death or serious injury arising from a fall and the risk increases with age. For example, in the US, 20 to $30 \%$ of older people who fall suffer moderate to severe injuries such as bruises, hip fractures, or head trauma (WHO). An estimated $95 \%$ of hip fractures are due to falls [16]. However, data on the prevalence and incidence of falls among the elderly are heterogeneous as there is no international consensus for assessing the fall risk profile of older people even for people at high risk of falls. People aged 75 or older, those who have fallen during the previous 12 months or those who have fear of falling or significant gait, muscle strength, or balance problems constitute the group of highest fall risk. The fall risk profile is also dependent on the setting and some other factors, including cognitive impairment. Even though a decline in balance, gait and muscle function increase the risk of falling, the 
relationship is not completely linear since those with most problems (i.e. bedridden) usually have a lower falls risk, similar to those without such problems, presumably due to low exposure to risk [2].

Knowledge of factors that predispose older persons to falls is important for tailoring appropriate preventive interventions. This is covered in more depth in Chap. 16.

\subsection{Incidence of Fragility Fractures}

Osteoporosis is clinically significant in the fractures that occur as a consequence of increased bone fragility. Although low BMD identifies individuals at increased risk of fracture, the majority of fragility fractures occur in individuals who have less marked reductions in bone mass or normal BMD, which means that other factors independent of BMD, such as the geometric and microarchitectural properties of the bone itself, and an individual's clinical risk factors, contribute to fracture risk [13]. The definition of an osteoporotic fracture is not straightforward, opinions differ concerning the inclusion or exclusion of different sites of fracture (most common are the forearm, hip, spine, proximal humerus) of fracture and also possible causative mechanisms [2]. One approach is to consider all fractures from low energy trauma as being osteoporotic. Low energy trauma is defined as a fall from a standing height or less, or trauma that in a healthy individual would not result in fracture [16]. The rising incidence of fractures with age might be caused by the rising incidence of falls and be multifactorial. The approach used in the report "Osteoporosis in the European Union" published by E. Hernlund and group in 2013 by and elsewhere is to characterise fracture sites as osteoporotic when they are associated with low bone mass and their incidence rises with age after of 50 years of age [2].

As hip fracture patients are treated in hospitals, the reports of incidence of hip fracture are more commonly available than reports of other sites of fractures [2]. Identifying the number of individuals at high fracture risk has value for future health resource allocation. Information on the incidence of fragility fractures varies between EU countries, as well as worldwide (Fig. 2.1). According to the calculations of the International Osteoporosis Foundation [2], there was marked heterogeneity in hip fracture risk between EU 27 countries: in women, the lowest annual incidences were found in Romania and Poland with the highest rates observed in Denmark and Sweden. There was an approximately three-fold range in hip fracture incidence which is somewhat lower than the tenfold range worldwide, but still substantial. The reason for the variation in hip fracture rates between countries is not known. Socio-economic prosperity seems to be one of the influencing factors, with higher hip fracture rates in countries with higher GDP.

In developing populations, however, particularly in Asia, the rates of osteoporotic fracture appear to be increasing $[8,13]$. The lifetime risk for hip, vertebral and forearm (wrist) fractures has been estimated to be approximately $40 \%$, similar to that for coronary heart disease. 
It has been estimated that in 2010 there were 21 million men and 137 million women aged 50 years or more at high fracture risk and that this number is expected to double by 2040, with the increase predominantly borne by Asia [13].

\subsection{Hip Fracture}

Hip fractures are an important cause of disability and death around the world, especially amongst older people. Studies have shown heterogeneity in annual agestandardised hip fracture rates globally (Fig. 2.1). The systematic review, published in 2012, used a literature survey covering a 50-year period and UN data on population demography [4]. By this review, the highest annual age-standardised hip fracture incidences (per 100,000 person-years) were observed in Scandinavia: Denmark (574), Norway (563) and Sweden (539), also in Austria (501). The lowest rates were detected in Tunisia (58) and Ecuador (73). North-Western Europe, Central Europe, the Russian Federation and Middle-East countries (Iran, Kuwait, Oman), Hong Kong, Singapore and Taiwan were high-risk countries for the hip fracture. Low-risk regions included Latin America (except for Argentina), Africa, and Saudi Arabia. There was around a tenfold range in hip fracture incidence worldwide, with the overall age-standardised incidence in men being half that of women $[4,13]$.

The highest incidence of hip fracture was observed mainly in countries furthest from the equator and in countries with extensive coverage of the skin due to religious or cultural practices, suggesting that vitamin D status might be an important factor underlying this distribution [4].

The aforenamed systematic review reported the age-standardised annual incidence of hip fractures to be higher in Hong Kong, Japan, South Korea and Taiwan than in the United States and some European countries [4]. This is in contrast to a study two decades ago that showed the hip fracture incidence to be higher in the United States compared to Hong Kong [17]. For women, Taiwan was in the highincidence category (incidence >300/100,000), ranking number 9 among 61 countries/regions. Hong Kong, Japan and South Korea were in the medium-incidence category (200-300/100,000), ranking 23, 32 and 34, respectively. China was in the low-incidence category $(<200 / 100,000)$. For men, Japan, Korea and Taiwan were among the high-incidence countries $(>150 / 100,000)$, while China and Hong Kong were in the moderate-incidence category $(100-150 / 100,000)[4,8]$.

The reasons for the large variation in age- and sex-adjusted hip fracture incidence worldwide are not clear. Genetic differences, environmental factors and treatment of osteoporosis might have a role. Interestingly, people living in the Mediterranean area have a lower incidence of fractures. This seems to be attributable to several factors, particularly higher serum 25-hydroxy vitamin D (25OHD) levels and healthier lifestyle [18]. Recent research highlights the role for a Mediterranean diet since the dietary pattern is associated with lower inflammation levels, lower adiposity and decreased risk of falls, all these factors being important for the development of hip fracture [19]. 
The total number of persons affected by hip fractures may be increasing over time in the following years, mainly due to the progressive ageing of the population. According to a conservative estimate, the annual number of hip fractures will increase from 1.66 million in 1990 to 6.26 million in 2050 [13]. Hip fracture rates appeared to have plateaued or decreased during the last one to two decades in many developed countries, following a rise in preceding years; however, in the developing world, age- and sex-specific rates are still rising in many areas [2, 8, 13]. In studies reporting a lower incidence of age- and sex-specific incidence hip fracture over time, possible explanations seem to be a higher adherence to anti-osteoporotic medications as well as increased use of calcium and vitamin D supplementation, avoidance of smoking and alcohol, and more efficacious strategies for the prevention of falls [20].

Technical reasons, such as inaccurate coding and recording of fractures and poor access to medical services in some regions might be part of the explanation of high variability in hip fracture incidence. According to the Eastern European \& Central Asian Regional Audit: Epidemiology, Costs and Burden of Osteoporosis in 2010, in Georgia, $75 \%$ of patients with hip fracture are not hospitalised and, in Kazakhstan and Kyrgyzstan, 50\% are not hospitalised due to poor access to surgical services and non-affordable medical care [13].

In summary, with a few exceptions, age-specific incidence rates of hip fracture significantly rose in Western populations until 1980 with subsequent stability or even a decrease. In Western countries, the trends seem to be more pronounced in women than in men [16]. However, confirmation is needed, by further longitudinal studies (particularly in non-white populations) to clarify in which direction we are moving. Moreover, the mean age of sustaining a hip fracture is increasing, meaning that this event is increasingly associated with disability and mortality, possibly because patients have more medical comorbidities.

\subsection{Other Osteoporotic Fractures}

From an epidemiological perspective, distal radius fractures are typical of the paediatric population, up to $25 \%$ of fractures in children involve the distal end of the radius, in older people up to $18 \%$ of all fractures involve the wrist [21]. The most common distal forearm fracture is a Colles' fracture. This fracture lies within $2.5 \mathrm{~cm}$ of the wrist joint margin and is associated with dorsal angulation and displacement of the distal fragment of the radius. It may be accompanied by a fracture of the ulna styloid process [2]. Forearm fractures account for a greater proportion in younger adults than the elderly. Conversely, hip fractures are rare at the age of 50 years but become the predominant osteoporosis fracture from the age of 75 years. In women, the median age for distal forearm fractures is around 65 years and for hip fracture, 80 years [2].

Vertebral fractures are the most common single osteoporotic fractures worldwide, these occur in 30-50\% of people more than the age of 50 [22]. In contrast to hip fractures, many factors limit the availability of reliable information on their 
epidemiology: two-thirds to three-fourths of vertebral fractures are clinically silent and less than $10 \%$ require hospital admission, which itself may vary due to geographic differences in access to healthcare.

For example, in a study involving about 1000 hospitalised persons of older age, chest radiographs were assessed for various reasons. Of importance, moderate (25-40\% height loss) or severe ( $>40 \%$ height loss) vertebral fractures were scored in $14 \%$ women by trained radiologists in a reference centre, but only half were reported in the X-ray report by the local radiologists [23]. This is often due to the scarce knowledge among radiologists in recognising asymptomatic vertebral fractures, the lack of any relevant signs or symptoms and/or the presence of other medical conditions for which a chest/abdomen X-ray was done that requires more attention (e.g. pneumonia or cancer) [23]. However, asymptomatic vertebral fractures are of importance since they represent a potential risk factor for symptomatic fractures and they are associated with a higher rate of disability and mortality, particularly in those having secondary forms of osteoporosis, such as glucocorticoid= induced osteoporosis [23].

In Europe, studies in men and women aged more than 50 years report that the incidence of clinical vertebral fracture is higher in men than women under 55 years, but that the risk rises in women after the age of 60 years [24]. The age-specific incidence of radiographic vertebral fracture (symptomatic or not) is estimated to be 2-2.5-fold higher in women than in men, being significantly higher in Scandinavia than in other European countries. It is reported that the incidence of vertebral fractures is highest at T12 and L1, second highest at L2 and L3, and third highest at T7 through T9 and at L4 [24]. However, the age-adjusted incidence may vary substantially from country to country. Some of these differences may be due to different patterns of clinical presentation and differences in vertebral fracture ascertainment in the studies that were used for calculating the incidence, rather than true differences in fracture incidence across countries. For example, symptomatic vertebral fracture rates seem to be particularly high in the United States, but the populationbased study on which of these estimates are calculated included vertebral fractures discovered incidentally on lateral spine imaging obtained for other clinical reasons, not only for assessing vertebral fractures as was the case in European countries [25].

In terms of prevalence data, it is estimated that, in both men and women, prevalence linearly increases with age independently of country, with some data suggesting that almost half of very old people (i.e. ageing $>85$ years) are affected by a vertebral fracture [24].

\subsection{The Burden of Fragility Fractures}

Measurement of the burden of diseases and injuries is a crucial input into health policy. The burden of osteoporosis in the 27 EU countries in 2010 was characterised by 22 million women and 5.5 million men to have osteoporosis; and 3.5 million new fragility fractures were sustained, comprising 610,000 hip fractures, 520,000 
vertebral fractures, 560,000 forearm fractures and 1,800,000 other fractures [2]. The greatest burden of hip fractures around the world is expected to occur in East Asia, especially China [8].

Information on the burden of osteoporosis in Latin American countries is limited. When analysing data in four Latin American countries [26] more than 840,000 osteoporosis-related fractures were predicted to occur in 2018, amounting to a total annual cost of $\sim 1.17$ billion USD. The total projected 5-year cost was $\sim 6.25$ billion USD. Annual costs were highest in Mexico (411 million USD), followed by Argentina (360 million USD), Brazil (310 million USD), and Colombia (94 million USD). The average burden per 1000 at risk was greatest in Argentina (32,583 USD), followed by Mexico (16,671 USD), Colombia (8240 USD), and Brazil (6130 USD). In the following 5 years, about four and half million fractures are anticipated to occur in these four countries.

To control and prevent these fractures, stakeholders must work together to close the care gap, particularly in secondary prevention. Indeed, all international scientific bodies agree upon the clinical recommendations to close the gap after a hip fracture, mainly: communicating with patients about their risks of future fractures and how to prevent them, by offering osteoporosis medications, improving long-term treatment persistence and assessing the risk of falls in routine follow-up visits [26].

\subsection{The Costs and Social Impact of Hip Fracture}

Hip fractures constitute a significant public health problem worldwide, and is associated with high rates of disability and mortality. Since hip fracture incidence increases linearly with advancing age and older people are expected to represent a growing proportion of the worldwide population in the near future, the costs of hip fracture will increase. Overall, hip fracture seems to be comparable to other disease groups, such as cardiovascular, in terms of hospitalisation and rehabilitation. However, other social costs, due to the onset of new comorbidities, sarcopenia, poor quality of life, disability and mortality are probably greater. Hip fracture and its surgical treatment predispose frail older persons to decompensation of chronic diseases, as well as complications such as anaemia, pneumonia, delirium, UTI and thromboembolic events.

Patients who have a hip fracture are at considerable risk for premature death. There has been no change in mortality rates for hip fractures since the last three decades, despite advancements in surgical solutions and the fact that the majority of patients in the developed economies are now operated on [27]. A report of osteoporosis in the European Union estimated that mortality related to low-impact trauma hip fracture is greater than road traffic accidents and equivalent to breast cancer [2]. The increased mortality risk after the hip fracture may persist for several years thereafter [28]. Patients experiencing hip fractures after low-impact trauma are at considerable risk for subsequent osteoporotic fractures and premature death [28]. 
In contrast to other types of fragility fracture (e.g. vertebral), hip fractures usually need immediate intervention and consequent hospitalisation. The mean duration of hospitalisation is highly variable dependent on local healthcare systems and populations studied.

A recent systematic review [29] has found that costs during the first year after the hip fracture $(\$ 43,669)$ are greater than equivalent estimates for the acute coronary syndrome $(\$ 32,345)$ and ischaemic stroke $(\$ 34,772)$. A systematic review of the costs of fragility hip fractures and key drivers of the differences in hip fracture costs between 1990 and 2015 analysed data of 670,173 patients from 27 different countries (mostly North America and Western Europe) [29]. The estimate for total health and social care costs in the first year following hip fracture was $\$ 43,669$ per patient. High variability was found between regions but also inside the country: in the United States, the costs of medical care in the 12 months ranged from $\$ 21,259$ to $\$ 44,200$. Inpatient care was with the highest cost, estimated at $\$ 13,331$. The mean length of stay for the index hospitalisation was 8.6 days and varied significantly by region. Studies from North America reported the shortest mean length of stay, whilst studies from Asia had the longest length of stay. Inpatient cost was followed by the cost of rehabilitation care, estimated at $\$ 12,020$. The high costs of rehabilitation might be biased as a limited number of studies in this category were available with a very high cost from one study [29].

Comorbidities prior to fracture were associated with significantly higher costs in $80 \%$ of studies. Developing a complication after hip fracture was associated with significantly higher costs in $93 \%$ of studies assessing this variable. Gender, year of study, US studies and length of stay were significantly associated with costs. In studies outside the United States, the mean cost per patient was \$3304 less per additional day of length of stay compared to the United States. On average, cases in females were found to be statistically significantly less expensive than in men, costing \$134 less per patient. Studies published more recently were significantly associated with lower costs: authors suggested that this might represent changes in clinical practice and fewer complications, as well as methodological changes [29].

Saving hospital costs by reducing the length of stay could be an important factor to diminish the expenditure on hip fracture management. Data from the Swedish National Patient Register, published in 2015, indicate that shorter length of hospital stay was associated with an increased risk of death after discharge in older hip fracture patients (mean age 82 years) with the length of stay 10 days less. In this study, the discharge placement was not available, but the authors suggest that the quality of rehabilitation in the early postoperative period might have influenced the outcome as reductions in length of stay will probably result in more complications occurring after discharge. Furthermore, shorter length of stay reduces the time available for the comprehensive geriatric assessment (CGA) during hospital stay [30]. CGA has been shown to decrease the risks of complications and death after discharge in elderly hip fracture patients.

In contrast, a study in the United States by Nikkel et al. demonstrated that decreased length of stay was associated with reduced rates of early mortality [31]. A total of 188,208 patients admitted to hospital for hip fracture in New York state 
from 2000 to 2011 , with 169,258 treated surgically and 18,950 treated nonsurgically, were analysed. The average length of hospital stay was 8.1 days; during the study, the average length of stay decreased from 12.9 days in 2000 to 5.6 days in 2011. The 30-day mortality rate for surgically-treated hip fracture patients was $4.5 \%$. A shorter hospital stay ( $<5$ days and $<10$ days) was associated with decreased 30-day mortality. Factors associated with increased 30-day mortality were nonsurgical treatment, male sex, being white, older age, longer time to surgery, blood transfusion, comorbid conditions, and discharge to hospice. The important factor in predicting early mortality is a longer time to surgery (more than $24 / 48 \mathrm{~h}$ after the fracture), which is also associated with a longer hospital stay, as has been described in earlier studies [32].

It has been estimated earlier that the expenditure needed for hip fracture exceeds that for breast and gynaecological cancers combined, but not those for cardiovascular disease in the United States [33]. The comparison of costs between hip fractures and cardiovascular diseases is intriguing. In Switzerland, osteoporotic hip fractures accounted for more hospital bed days than myocardial infarction and stroke and consequently led to higher costs [34], while in Italy the costs due to hip fractures were comparable to those of acute myocardial infarction.

As described in Chap. 12, rehabilitation is crucial for people after hip fracture [35]. However, the advanced age and the comorbidities affecting hip fracture patients often dictate that the completion of the rehabilitation programme takes place in long-term care (LTC) facility or a nursing home. The costs needed for a LTC seem to be almost double those required by a rehabilitation institute. However, the roles of these organisations for the rehabilitation of older patients are still debated. In a well-known study on this topic, hip fracture patients admitted to rehabilitation hospitals did not differ from patients admitted to nursing homes in their return to the community or disability rate [36]. Moreover, costs were significantly greater for rehabilitation hospital patients than for nursing home patients and the evidence about the value of these organisations in the elderly is still conflicting.

Poor recovery among older adults with hip fractures can occur despite successful surgical repair and rehabilitation, suggesting other factors might play a role in recovery, such as social factors [37]. In the review of the role of social factors in recovery after hip fracture, the majority of included studies have shown that both a higher level of social support and a better socioeconomic status has a positive effect on physical functional recovery post-hip fracture in individuals aged more than 65 years. Socioeconomic status was associated with both physical functional recovery and mortality from hip fractures. Income, employment, education skills and training are all socioeconomic factors that have been studied and found to influence recovery from hip fracture [7, 37].

Other consequences may be loss of muscle strength, increased postural sway and decline in walking speed that can lead to loss of functional muscle mass, sarcopenia and finally to disability. The impact on disability is striking: 1 year after fracturing a hip, $40 \%$ of patients are still unable to walk independently, $60 \%$ have difficulty with at least one essential activity of daily living, and $80 \%$ are restricted in instrumental activities of daily living, such as driving and grocery shopping [16]. 
A review published in 2016 included data from 32 cohort studies mostly from Western European and North American countries, but also included some studies from Australia, New Zealand, Japan and China [37]. The review provided clear evidence that people recovering from hip fractures experience ongoing limitations in mobility, basic activities of daily living (ADL), self-care, participation and quality of life. Between 40 and $60 \%$ of hip fracture survivors are likely to recover their pre-fracture level of mobility. Up to $70 \%$ of people may regain their pre-fracture level of independence for composite measures of basic ADL, but this proportion is likely to be lower for those with higher levels of dependence pre-fracture [37]. Only half or fewer people experiencing hip fracture may regain their pre-fracture level of independence in instrumental activities of daily living (IADLs). Most people who recover their ability to perform basic or instrumental ADLs do so within the first 6 months after discharge, although the time to recovery for individual ADLs ranges from approximately 4 to 11 months [37]. Studies in many countries world-wide have indicated that hip fracture has a significant impact on the quality of life in the medium to long term. In Western nations, between 10 and $20 \%$ of hip fracture patients are institutionalised within 6-12 months post-fracture [37].

Hip fracture seems to be associated with the onset of other co-morbidities with a high cost for society. Recent research has highlighted that people experiencing a hip fracture have a greater incidence of depression [38] and consequently a higher use of anti-depressant medications [39]. Another field of interest is the possible relationship between hip fracture and the onset of cardiovascular diseases. Hip fracture, in fact, seems to increase the risk of coronary heart disease, particularly during the first year after the event [40]. Since cardiovascular diseases are among the most expensive medical conditions, the impact of hip fracture in contributing to a huge increase in medical and social costs is highly relevant.

\subsection{The Costs and Social Impact of Other Osteoporotic Fractures}

Other osteoporotic/fragility fractures (e.g. wrist, spine, shoulder) are more common than hip fractures, but some of them (such as vertebral) are often asymptomatic and the others require less hospital stay than hip fractures. Limited data exist regarding the social costs of the other osteoporotic fractures. In a study including six different cohorts, the authors report that the adjusted mean first-year costs associated with hip, vertebral, and non-vertebral fractures were $\$ 26,545, \$ 14,977$, and $\$ 9183$ for the 50-64 age cohort, and $\$ 15,196, \$ 6701$, and $\$ 6106$ for patients $\geq 65$ years, respectively [41]. However, after considering the prevalence rate of all major osteoporotic fractures, the proportion of the total fracture costs accounted for by non-vertebral, hip, and vertebral fractures were $66 \%, 21 \%$ and $13 \%$ in younger and $36 \%, 52 \%$ and $12 \%$ for in older people [41].

The importance of osteoporotic fractures, other than hip, from an economic point of view, was confirmed by a large study using administrative data and including almost all European countries. In this report, European countries report a marked 
difference in the LOS between men and women, with differences ranging from 0.32 days in Austria to 20.2 days in Spain [42]. The total cost of vertebral fractures in the European Union is enormous, being estimated at 377 million euros per yearabout $63 \%$ of hip fractures [42]. However, more economic data are needed for nonfemoral fractures, including data regarding asymptomatic fractures; this will require cohort studies designed to achieve this aim.

\subsection{Conclusions}

Hip fracture is a common and debilitating condition, particularly in older persons. Although the age- and gender-specific incidence is decreasing in some countries, the global incidence of hip fracture is rising worldwide, due to population ageing. More attention should be paid to prevention, given its great impact on social costs and quality of life. Other osteoporotic fractures, in particular those affecting spine and wrist, play an important role from an epidemiological point of view, but more data on their economic impact are needed. Thus, further epidemiological studies are needed to better verify the trends in the incidence of osteoporotic fractures and the strategies of effective prevention.

\section{References}

1. WHO. https://www.who.int/news-room/fact-sheets/detail/ageing-and-health. Approached February 1, 2020

2. Hernlund E, Svedbom A, Ivergård M, Compston J, Cooper C, Stenmark J, McCloskey EV, Jönsson B, Kanis JA (2013) Osteoporosis in the European Union: medical management, epidemiology and economic burden. A report prepared in collaboration with the International Osteoporosis Foundation (IOF) and the European Federation of Pharmaceutical Industry Associations (EFPIA). Arch Osteoporos 8:136

3. Zanker J, Duque G (2019) Osteoporosis in older persons: old and new players. J Am Geriatr Soc 67(4):831-840

4. Kanis JA, Oden A, McCloskey EV et al (2012) A systematic review of hip fracture incidence and probability of fracture worldwide. Osteoporos Int 23(9):2239-2256

5. Liu J, Curtis EM, Cooper C, Harvey NC (2019) State of the art in osteoporosis risk assessment and treatment. J Endocrinol Investig 42(10):1149-1164

6. Veronese N, Maggi S (2018) Epidemiology and social costs of hip fracture. Injury 49(8): $1458-1460$

7. Auais M, Al-Zoubi F, Matheson A, Brown K, Magaziner J, French SD (2019) Understanding the role of social factors in recovery after hip fractures: a structured scoping review. Health Soc Care Community 27(6):1375-1387

8. Cheung EYN, Tan KCB, Cheung CL, Kung AWC (2016) Osteoporosis in East Asia: current issues in assessment and management. Osteoporos Sarcopenia 2:118-133

9. Yu F, Xia W (2019) The epidemiology of osteoporosis, associated fragility fractures, and management gap in China. Arch Osteoporos 14(1):32

10. Khadilkar AV, Mandlik RM (2015) Epidemiology and treatment of osteoporosis in women: an Indian perspective. Int J Women's Health 7:841-850

11. Francisco L, Délio Marques C, Costa-Paiva L, Pinto-Neto AM (2015) The epidemiology and management of postmenopausal osteoporosis: a viewpoint from Brazil. Clin Interv Aging 10:583-591 
12. Wright NC, Looker AC, Saag KG et al (2014) The recent prevalence of osteoporosis and low bone mass in the United States based on bone mineral density at the femoral neck or lumbar spine. J Bone Miner Res 29(11):2520-2526

13. Curtis EM, Moon RJ, Harvey NC, Cooper C (2017) The impact of fragility fracture and approaches to osteoporosis risk assessment worldwide. Bone 104:29-38

14. Cruz-Jentoft A, Landi F, Schneider FM et al (2014) Prevalence of and interventions for sarcopenia in ageing adults: a systematic review. Report of the International Sarcopenia Initiative (EWGSOP and IWGS). Age Ageing 43(6):748-759

15. Huo YR, Suriyaarachchi P, Gomez F, Curcio CL, Boersma D, Muir SW, Montero-Odasso M, Gunawardene P, Demontiero O, Duque G (2015) Phenotype of osteosarcopenia in older individuals with a history of falling. J Am Med Dir Assoc 16:290-295

16. Cooper C, Cole ZA, Holroyd CR, Earl SC, Harvey NC, Dennison EM et al (2011) Secular trends in the incidence of hip and other osteoporotic fractures. Osteoporos Int 22(5):1277-1288

17. Ho S, Bacon E, Harris T, Looker A, Maggi S (1993) Hip fracture rates in Hong Kong and the United States, 1988 through 1989. Am J Public Health 83:694-697

18. Haring B, Crandall CJ, Wu C, LeBlanc ES, Shikany JM, Carbone L et al (2016) Dietary patterns and fractures in postmenopausal women: results from the women's health initiative. JAMA Intern Med 176(5):645-652

19. Romero Pérez A, Rivas VA (2014) Adherence to Mediterranean diet and bone health. Nutr Hosp 29(5):989-996

20. Brauer CA, Coca-Perraillon M, Cutler DM, Rosen AB (2009) Incidence and mortality of hip fractures in the United States. JAMA 302(14):1573-1579

21. Nellans KW, Kowalski E, Chung KC (2012) The epidemiology of distal radius fractures. Hand Clin 28(2):113-125

22. Ballane G, Cauley JA, Luckey MM, Fuleihan GE (2017) Worldwide prevalence and incidence of osteoporotic vertebral fractures. Osteoporosis Int 28:1531-1542

23. Lems WF (2007) Clinical relevance of vertebral fractures. Ann Rheum Dis 66(1):2-4

24. Ensrud KE, Schousboe JT (2011) Vertebral fractures. NEJM 364(17):1634-1642

25. Amin S, Achenbach SJ, Atkinson EJ, Khosla S, Melton LJ III (2014) Trends in fracture incidence: a population-based study over 20 years. J Bone Miner Res 29(3):581-589

26. Aziziyeh R, Amin M, Habib M, Perlaza JG, McTavish RK, Lüdke A, Fernandes S, Sripada K, Cameron C (2019) A scorecard for osteoporosis in four Latin American countries: Brazil, Mexico, Colombia, and Argentina. Arch Osteoporos 14(1):69

27. Johansen A, Golding D, Brent L, Close J, Gjertsen J-E, Holt G, Hommel A, Pedersen AB, Röck ND, Thorngren K-G (2017) Using national hip fracture registries and audit databases to develop an international perspective. Injury 48(10):2174-2179

28. Abrahamsen B, van Staa T, Ariely R, Olson M, Cooper C (2009) Excess mortality following hip fracture: a systematic epidemiological review. Osteoporos Int 20(10):1633-1650

29. Williamson S, Landeiro F, McConnell T et al (2017) Costs of fragility hip fractures globally: a systematic review and meta-regression analysis. Osteoporos Int 28:2791-2800

30. Nordstrom P, Gustafson Y, Michaelsson K, Nordstrom A (2015) Length of hospital stay after hip fracture and short term risk of death after discharge: a total cohort study in Sweden. BMJ 350:h696

31. Nikkel LE, Kates SL, Schreck M, Maceroli M, Mahmood B, Elfar JC (2015) Length of hospital stay after hip fracture and risk of early mortality after discharge in New York state: retrospective cohort study. BMJ 351:h6246

32. Maggi S, Siviero P, Wetle T, Besdine RW, Saugo M, Crepaldi G (2010) A multicenter survey on profile of care for hip fracture: predictors of mortality and disability. Osteoporosis Int 21(2):223-231

33. Hoerger TJ, Downs KE, Lakshmanan MC, Lindrooth RC, Plouffe L, Wendling B et al (1999) Healthcare use among U.S. women aged 45 and older: total costs and costs for selected postmenopausal health risks. J Womens Health Gend Based Med 8(8):1077-1089

34. Lippuner K, von Overbeck J, Perrelet R, Bosshard H, Jaeger P (1997) Incidence and direct medical costs of hospitalizations due to osteoporotic fractures in Switzerland. Osteoporosis Int 7(5):414-425 
35. De Rui M, Veronese N, Manzato E, Sergi G (2013) Role of comprehensive geriatric assessment in the management of osteoporotic hip fracture in the elderly: an overview. Disabil Rehabil 35(9):758-765

36. Kramer AM, Steiner JF, Schlenker RE, Eilertsen TB, Hrincevich CA, Tropea DA et al (1997) Outcomes and costs after hip fracture and stroke. A comparison of rehabilitation settings. JAMA 277(5):396-404

37. Dyer SM, Crotty M, Fairhall N, Magaziner J, Beaupre LA, Cameron ID, Sherrington C (2016) Fragility Fracture Network (FFN) Rehabilitation Research Special Interest Group. A critical review of the long-term disability outcomes following hip fracture. BMC Geriatr 16(1):158

38. Cristancho P, Lenze EJ, Avidan MS, Rawson KS (2016) Trajectories of depressive symptoms after hip fracture. Psychol Med 46(07):1413-1425

39. Iaboni A, Seitz DP, Fischer HD, Diong CC, Rochon PA, Flint AJ (2015) Initiation of antidepressant medication after hip fracture in community-dwelling older adults. Am J Geriatr Psychiatry 23(10):1007-1015

40. Veronese N, Stubbs B, Crepaldi G, Solmi M, Cooper C, Reginster J-Y et al (2017) Low bone mineral density and fractures are associated with incident cardiovascular disease: a systematic review and meta-analysis. Osteoporosis Int 28:180-181

41. Shi N, Foley K, Lenhart G, Badamgarav E (2009) Direct healthcare costs of hip, vertebral, and non-hip, non-vertebral fractures. Bone 45(6):1084-1090

42. Finnern HW, Sykes DP (2003) The hospital cost of vertebral fractures in the EU: estimates using national datasets. Osteoporosis Int 14(5):429-436

Open Access This book is licensed under the terms of the Creative Commons AttributionNonCommercial-NoDerivatives 4.0 International License (http://creativecommons.org/licenses/ by-nc-nd/4.0/), which permits any noncommercial use, sharing, distribution and reproduction in any medium or format, as long as you give appropriate credit to the original author(s) and the source, provide a link to the Creative Commons license and indicate if you modified the licensed material. You do not have permission under this license to share adapted material derived from this book or parts of it.

The images or other third party material in this book are included in the book's Creative Commons license, unless indicated otherwise in a credit line to the material. If material is not included in the book's Creative Commons license and your intended use is not permitted by statutory regulation or exceeds the permitted use, you will need to obtain permission directly from the copyright holder.

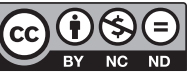

